\title{
ACADEMIA
}

Accelerating the world's research.

\section{Impact of wavelength converters in wavelength routed all-optical networks}

ezhil rajan

Computer Communications

\section{Cite this paper}

Get the citation in MLA, APA, or Chicago styles

\section{Downloaded from Academia.edu $\pi$}

\section{Related papers}

A Heuristic for Placement of Limited Range Wavelength Converters in All-Opt ical Networks shiva kumar

Dynamic rout ing and wavelength assignment algorithms in wavelength division mult iplexed transluce...

Chao-Chin Lu

Converter placement in all-optical networks using genetic algorithms shiva kumar 


\title{
computer communications
}

\section{Impact of wavelength converters in wavelength routed all-optical networks}

\author{
K.R. Venugopal, E. Ezhil Rajan, P. Sreenivasa Kumar* \\ Department of Computer Science and Engineering, Indian Institute of Technology, Chennai 600 036, India
}

Received 27 July 1998; accepted 19 October 1998

\begin{abstract}
This paper attempts to study the impact of wavelength converters in WDM wavelength routed all-optical networks. A new heuristic approach for placement of wavelength converters to reduce blocking probabilities is explored. Multihop virtual topology is designed to minimize the number and overall cost of the converters. Blocking probabilities for Static Lightpath Establishment (SLE) and Dynamic Lightpath Establishment (DLE) are analyzed. In the case of SLE, arranging lightpaths in ascending order of their path length reduces blocking probability. Wavelength converters placed at nodes with high nodal degree further reduces the blocking probabilities. Simulation studies performed on 28-node USA long haul network, 20-node arbitrary mesh network, and 19-node EON (European Optical Network) validate the observations made earlier. (C) 1999 Elsevier Science B.V. All rights reserved.
\end{abstract}

Keywords: Wavelength converters; Wavelength routed all-optical networks; Multihop virtual topology

\section{Introduction}

Wavelength Division Multiplexing (WDM) wavelength routed all-optical WANs have emerged as the most efficient means to meet the ever increasing integrated demands of the communication applications. The advantages of WDM optical networks over conventional networks is large bandwidth, scalability, low power loss, modularity, reliability, reconfigurability and protocol transparency. The wavelength routed networks, have potential to meet the exponential growth in the user traffic catering to the needs of divergent requirements such as high bandwidth online medical applications, real-time rocket and satellite communications, secure transaction processing in federated databases, defense applications, multimedia traffic, supercomputer inter-connects along with smaller bandwidth requirements of voice, data and many other applications to millions of users [31,33].

The inability of the end users to generate data more than a few gigahertzs (electronic bottleneck) and the enormous bandwidth of the fiber has led to the development of WDM technology. The optical transmission bandwidth is split into a number of distinct non-interfering and non-overlapping wavelength communication channels each operating on a different optical wavelength and at different bit

\footnotetext{
* Corresponding author. Tel.: + 91 442351365. Ext. 3501; fax: + 9144235 0509; e-mail: psk@iitm.ernet.in
}

rates (peak electronic rate of $\mathrm{Gb} / \mathrm{s}$ ) [29]. The co-existence of a number of channels on a single fiber increases the utilization of the fiber bandwidth throwing challenges to design and develop suitable network architectures, protocols and algorithms [15].

This paper attempts to study the performance of wavelength converters in WDM wavelength routed optical networks for SLE and DLE. Heuristics for better wavelength converter placement and for ordering lightpaths requests in Static Lightpath Establishment problem are proposed and validated in this paper. Section 2 presents the network model, while Section 3 describes the wavelength converter architecture and its components. A review of related work is presented in Section 4. Section 5 addresses the wavelength converter problem. An analytical model for minimum wavelength conversion cost is given in Section 6. Section 7 provides the algorithm for SLE and DLE respectively. Simulation and numerical results are covered in Section 8. Concluding remarks are presented in Section 9.

\section{Network model}

The network model consists of a number of access stations attached to a group of wavelength routing optical nodes. Each access station has at least one tunable optical transmitter and one tunable optical receiver as well as 


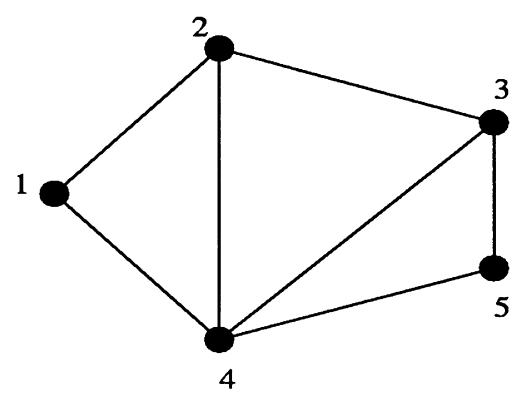

Fig. 1. Physical topology.

opto-electronic interface to the user(s). Each optical link consists of a pair of unidirectional optical fibers thereby providing a bidirectional path. The optical transmitter/ receiver defines the boundary of the optical layer. The signals inside the network are in the optical domain and signals outside the network are in electronic domain [24]. The physical topology of the WDM optical networks consists of wavelength routing nodes, interconnected by pairs of point-to-point fiber links in an arbitrary topology (Fig. 1).

Wavelengths are assigned to the transmitters and receivers creating several independent channels all of which are multiplexed onto the optical fiber defining the logical connectivity among the nodes. By changing the wavelength assignment, the resulting connection graph can be adapted to the changing traffic patterns, station failures and station additions. In this architecture, the use of wavelength multiplexers provide the advantage of higher aggregate system capacity owing to spatial reuse of wavelengths and support a large number of users even with a limited number of wavelengths. It is claimed that WDM optical networks can support a hundred million nodes giving a throughput of ten million terabits with just eight wavelengths [3].

A lightpath consists of a path through the network between nodes and a wavelength on each of these links in that path and does not require electronic processing at the

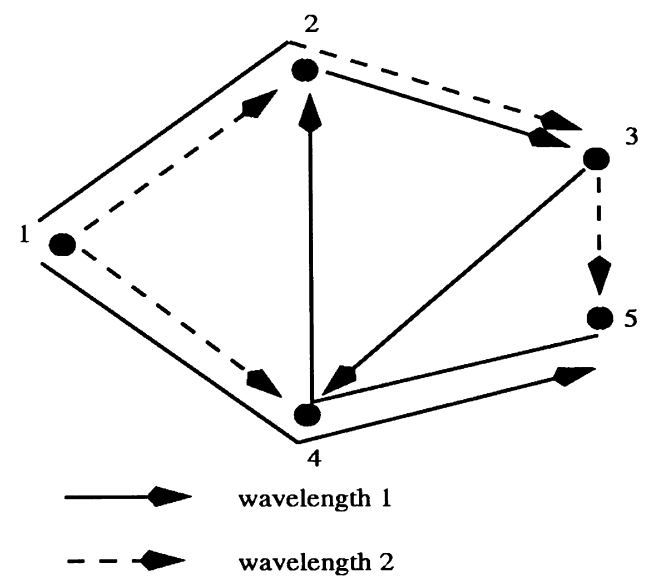

Fig. 2. Virtual topology. intermediate nodes. Lightpaths are setup by configuring the nodes in the networks. Some or all of the routing nodes (crossconnect nodes) have the ability to terminate the lightpaths. A lightpath provides a path between nodes with a bandwidth equal to that of one channel. Each lightpath may be used to carry either circuit switched or packet switched data independent of other lightpaths in the fiber. This property is called optical transparency. The set of all lightpaths that were setup between nodes constitutes the virtual topology [34]. Virtual topology for the physical topology with the set of lightpaths in Fig. 1 is shown in Fig. 2. The virtual topology is a graph with the nodes corresponding to the nodes in the network with an edge from node $x$ to node $y$ if a lightpath is set up from node $x$ to node $y$.

In Fig. 2, the lightpaths established between 1-5 and 2-5 do not require any processing at the node 4 . However, the same wavelength can be reused by the disjoint lightpaths within the optical network (wavelength reuse). The wavelength 2 is used between nodes, 2-3, 3-5, 1-2, and 1-4.

It is usually not feasible to establish lightpaths between every pair of nodes owing to physical constraints on the number of wavelengths limited by the erbium doped fiber amplifier bandwidth $(35 \mathrm{~nm})$ [12], limited number and tunability of optical receivers at each node and lightwave dispersions that limit the physical length of the lightpath. In general, a single optical wavelength may not be available between a given source and destination. It is possible to remove the wavelength continuity constraint by employing wavelength conversion at the intermediate nodes. Therefore, a transmission path is obtained by setting up and chaining several lightpaths to establish connection between source-destination pairs. In Fig. 2, the lightpath between nodes 1-3 undergoes wavelength conversion at node 2 from $\lambda_{1}$ to $\lambda_{2}$. When wavelengths are assigned dynamically, the wavelength continuity constraint blocks new lightpath requests even though wavelengths are available on the fiber links between the source and destination.

A switch is an important component of the optical network. It routes an incoming signal to an outbound port without any opto-electronic conversion. In a non-reconfigurable switch, the outgoing routes for different signals arriving at any input port is fixed. In a reconfigurable switch, signals on a wavelength $\lambda_{i}$ propagates through an optical switch which outputs them in a specific permutation independent of all other wavelengths; and unlike in a non-reconfigurable switch, this permutation can be set for each wavelength.

\section{Wavelength converters}

\subsection{Architecture}

A wavelength converter can transfer a signal on one wavelength to another wavelength at its output port. A 


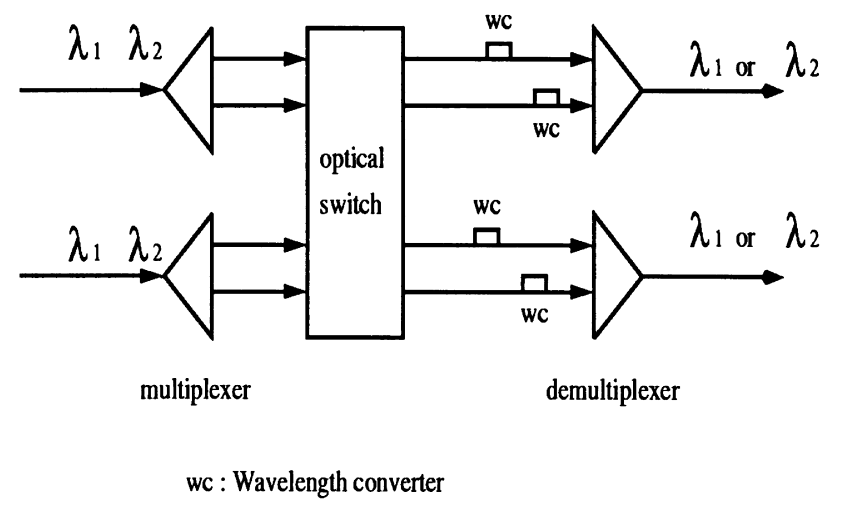

Fig. 3. Wavelength switch and converter.

group of wavelength converters is called wavelength converter bank. A wavelength converter switch can route a signal from any input port to any output port on different wavelengths provided it does not conflict with the signals on that port (Fig. 3).

\subsection{Methods of wavelength conversion}

Wavelength conversion methods can be grouped into two types: Opto-electronic conversion and all-optical conversion. In the former, wavelength conversion is achieved by transforming the optical signal to electronic signal and converting it back to the optical signal on a different wavelength. Wavelength conversion uses a back to back connection of a photodetector and a laser where the optical signal is converted into an electrical signal and drives the output of a tunable laser. However, this introduces opto-electronic conversion delay and decreases the network speed. In the latter, the signal remains completely in the optical domain. The all-optical conversion methods can be further grouped into two categories: those which employ coherent effects such as Four Wave Mixing (FWM) and Difference Frequency Generation (DFG) and those which use crossmodulation such as absorption saturation of the semiconductor laser diode and gain Saturation of Optical Amplifier (SOA) [25].

Wavelength converters employing the concept of Four Wave Mixing (FWM) has limited range convertibility and perform better than those employing wavelength converters with full range conversions [14]. Saturated Optical Amplifiers [7] based on cross modulation technique has lower distortion when converted to a higher frequency than to a lower frequency. The quality of the signal deteriorates when it passes through cascaded conversions and therefore the

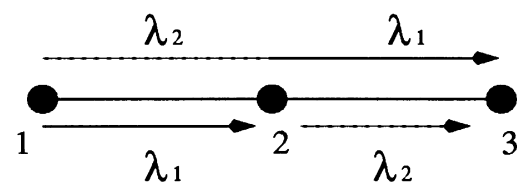

Fig. 4. Wavelength conversion at node 2. number of cascaded conversions should be limited before the signal reaches the destination [31]. FWM is efficient in terms of power maintenance and to convert to wavelengths that are closer (in terms of $\mathrm{nm}$ ) than those that are farther away. A wavelength converter must be protocol transparent, have low setup time, large signal-to-noise ratio (SNR), flexibility to convert both to shorter and longer wavelengths, low chirp signal to high extinction ratio and simple to implement [2].

\subsection{Conversion degree of a wavelength converter}

At any node, wavelength conversion capability, can be classified by a conversion degree $d$. Let $\mathrm{w}$ represent the number of wavelengths on a fiber link. If an incoming wavelength $\lambda_{i}$ can be converted to some fixed outgoing wavelength $\lambda_{j}$, then it corresponds to fixed conversion and $d=1$ ( $\lambda_{i}=\lambda_{j}$ corresponds to no wavelength conversion). A wavelength $\lambda_{i}$ can be converted to any one of the wavelength $\lambda \in r(i)$ where $|r(i)|=d \leq w$. If $d<w$, then it corresponds to limited range wavelength conversion, and $d=w$ corresponds to full range wavelength conversion [32].

\subsection{Limited wavelength conversion}

Limited wavelength conversion reduces the hardware costs of the optical switching nodes. With the increased cost and complexity of the optical node with wavelength converters and a limited range of conversion (as in FWM), a limited amount of wavelength conversion may be economical [26]. Theoretical studies show that most of the reduction in the blocking probabilities is achieved using limited conversion. Sparse or limited wavelength conversion can be of three types:

- a limited number of nodes are provided with full convertibility;

- a limited number of converters are placed at all the nodes (share-per-link and share-per-node); and

- a limited range of conversion is only possible at the nodes.

\subsection{Blocking probability}

Blocking probability is the ratio of the number of lightpath requests rejected to the number of lightpath connections requested. Fig. 4 shows the lightpaths established between nodes 1-2, 2-3 and 1-3. Each fiber supports two wavelengths, $\lambda_{1}$ and $\lambda_{2}$. A connection request is set up between nodes 1-2 on $\lambda_{1}$ and between nodes 2-3 on $\lambda_{2}$. A next connection request between nodes 1-3 can be set up only on a two-hop path; i.e. 1-2 on $\lambda_{2}$ and 2-3 on $\lambda_{1}$. This is possible only if the optical switching node has a wavelength converter at 2, otherwise, the call is blocked (Fig. 4). Thus nodes with wavelength conversion capability reduce blocking probability.

Consider a linear chain of $l$ hops as shown in Fig. 5. Let $w$ 


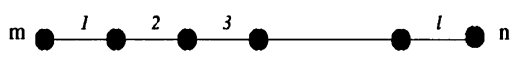

Fig. 5. A $l$ hop linear network.

be the number of wavelengths available on a fiber link, $x$ be the probability that a wavelength is used on a link, then $x^{w}$ represents the expected number of busy wavelengths. It is assumed that a wavelength used on a link is statistically independent of other links and other wavelengths.

In a network without wavelength converters at nodes, the probability $P_{n w c}$ that a connection request is blocked between $m$ and $n$ with $l$ hops is the probability that each wavelength of the wavelength set $w$ is used on at least one of the $l$ links [22].

$$
P_{n w c}=\left[1-(1-x)^{l}\right]^{w}
$$

In a network with wavelength converters at nodes, the probability $P_{w c}$, that a connection request is blocked between $m$ and $n$ with $l$ hops is the probability that all wavelengths of the wavelength set $w$ is used on at least one of the links.

$$
P_{w c}=1-\left(1-x^{w}\right)^{l}
$$

This suggests that the blocking probability with and without wavelength converters increase with the number of hops $l$. The blocking probability increases rapidly in the case of network without wavelength converters and hence it is necessary to keep the path length small, i.e., small network diameter. This is ensured by considering path length and congestion in designing a routing algorithm and including additional fibers between the nodes of the network without wavelength converters. In contrast, blocking probability is much lower in networks with wavelength converters and fiber utilization is higher, but fiber utilization decreases with the increase in the number of hops. Benefit of wavelength conversion increases with the length of the path and decreases with the increase in the number of wavelengths.

\subsection{Wavelength reuse}

A wavelength can be used in the disjoint paths of the

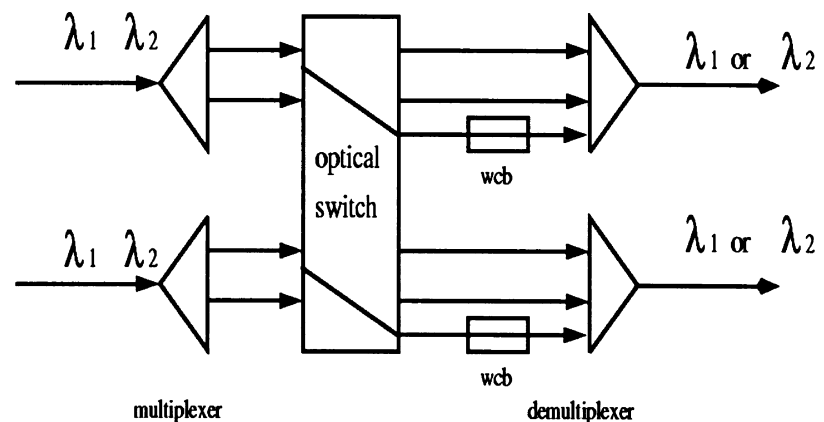

wcb: wavelength converter bank

Fig. 6. Share-per-link converters.

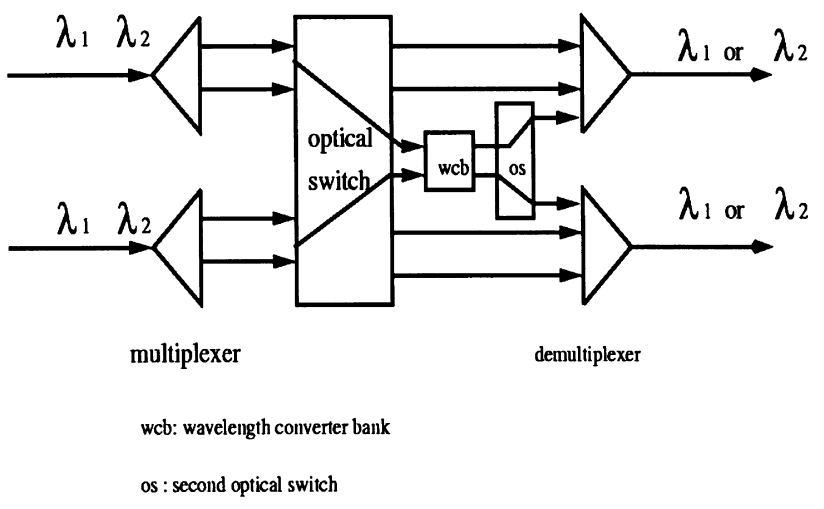

Fig. 7. Share-per node converters.

network and is termed as Wavelength Reuse. The concept of wavelength reuse factor $R$ can be defined as (Offered load)/(Number of wavelength in use). The offered load is the product of the arrival of the lightpath requests and the average lightpath duration. $R$ is dependent on four factors, namely, the network size and topology, the traffic distribution in the network, the number of wavelengths, and the routing and wavelength assignment algorithm [24]. The reuse factor increases with the network size $N$ since the average length of the lightpath increases as $\log N$ thereby giving a reuse factor approximately as $N / \log N$. The value of $R$ grows with the increase in the number of wavelengths and wavelength conversion. A particularly interesting analysis about reuse factor is available in [16,24]. In Fig. 4, it is obvious that three connections can be established by reusing the wavelengths if there is a converter at node 2. In Fig. 2, wavelength 2 was reused between nodes 2-3, 3-5, 1-2 and $1-4$.

\subsection{Load}

Given a set of lightpath requests and routing, the parameter Load $L$ is defined as the maximum over all links of the number of wavelengths in use on a link, i.e., $L=\max _{i} l_{i}$ where $l_{i}$ represents the number of lightpaths on a link. The maximum load that can be supported will be at most the number of wavelengths that are available on a link. It represents the utilization of the fiber links. The objective is to determine the maximum load that can be supported as a function of the number of wavelengths and the degree of wavelength conversion. In general, any arbitrary topology with limited conversion capability at optical nodes can support higher loads. Extensive study of load in ring networks is available in [17-20,23]. In the case of dynamic lightpath establishment, Load is the rate of connection requests per unit time. One Erlang is the number of calls per unit call holding time.

\subsection{Cost}

Wavelength converters can be dedicated to each channel (Fig. 3), or a group of channels can share a wavelength 
converter to reduce the cost, as some channels may not need wavelength converters. A dedicated converter bank can be provided to each link (Fig. 6) or to each node (Fig. 7). The signals which do not require conversion passes through the optical switch and then to the outbound link. The signals that require wavelength conversion are directed to the converter bank and the converted signal is forwarded to the outbound link.

Of the three configurations, dedicated channel converter is costlier, but easy to switch. The share-per-node is cheaper but increases the switching complexity as the signal passes through the optical switch before going to the wavelength converter bank and then again to another optical switch and finally to the outbound fiber. The share-per-link is a good trade-off when compared with the other two.

\subsection{Switch complexity}

The introduction of a large number of converters leads to a large, expensive and complex switches (a large switch tends to mix signals effectively lowering blocking probability). It is possible to operate a switch with only a few wavelength converters and still achieve most of the benefits of a switch that has full conversion [21].

\subsection{Delay}

There is some delay introduced in the optical conversion and the delay further increases if the conversion is optoelectronic conversion. The delay caused by the wavelength conversion may not be suitable for the real-time traffic. When a lightpath request arrives, a very small duration is necessary to compute the path and tune the wavelength converters at the intermediate nodes. After the initial setup, the data can propagate through the optical path without any further processing [11].

\subsection{Congestion, throughput, rerouting}

Congestion is the utilization of the heaviest loaded link in the network. Congestion is reduced and the network throughput increases. Rerouting becomes much easier in the case of network nodes equipped with wavelength converters and it becomes robust to channel, link and node failures [19].

\subsection{Network topology}

The benefits accrued by providing the optical nodes with wavelength converters depend on the number of wavelengths, network topology and the traffic matrix characteristics. The lower bound on the number of wavelengths required in a wavelength convertible network is reduced [31]. In dense networks, the effectiveness of the wavelength converters is limited [13], and it has no effect on the blocking performance in a completely connected network as all connections are single hop. In contrast, the converters in sparsely connected networks do not mix the connection requests well, causing load correlation in successive links.

\subsection{Wavelength assignment}

A small number of nodes equipped with wavelength convertibility ensures the desired performance. Wavelengths are assigned to the connection request randomly from the set of free wavelengths on the computed route. The link loads are independent and the wavelengths used on a link are uniformly distributed over the entire set of wavelengths independent of all the other links. It is assumed that a wavelength is used on a link with a fixed probability independent of other wavelengths and therefore the dynamic nature of the traffic is hidden. In light of all these advantages and constraints, it is necessary to optimize the number and placement of converters to ensure low blocking probability and effective utilization of the wavelength converters. Wavelength routed optical networks with wavelength converters are most likely to be used in WANs where a broadcast and select approach to switching ceases to feasible.

\section{Related work}

In [11], wavelength converters are employed to resolve wavelength conflicts and increase wavelength reuse. A shortest path routing algorithm is used to reduce the number of converters leading to the concept of share-per-node or share-per-link wavelength convertible switch. Blocking probability is reduced with the use of wavelength converters, but the complexity of the algorithm is $O\left(n^{4} w^{2}\right)$, where $n$ is the number of wavelength routers and $w$ is the number of wavelengths per fiber link. A faster algorithm of $O\left(n^{2} w^{2}\right)$ is developed in [4].

Barry et al. [21] have derived a lower bound on the number of switching states in a circuit switched network with wavelength converters. In [13], each switching node in the network has a limited number of wavelength converters to resolve wavelength conflicts in multihop paths. Wavelength converters cause an insignificant reduction in blocking probability at light loads whereas at medium loads, the gain is significant. Alternate routing with wavelength conversion reduces blocking probability, but introduces network instability [6].

In [16,24], lower bounds and approximate analytical models with and without wavelength converters are studied. The routing in optical networks is treated in the same manner as routing in circuit switched networks. Each switching node has full conversion capability and requires $d \times w$ wavelength converters with each switching node having $d$ ports and $w$ wavelengths per port. Employing a large number of wavelength converters is not cost effective. In [24], the RWA problem is formulated as ILP with the objective of maximizing the number of lightpaths that are successfully routed. The formulation is similar to the 
multicommodity flow problem with integer flows through the fiber links.

In [1], a generalized reduced load approximation scheme was used to calculate the blocking probabilities for the optical network employing fixed routing for arbitrary topology with paths lengths of at most three hops. A least loaded routing algorithm for fully connected networks with paths of one or two hops are also explored. It considers Poisson input traffic and uses a Markov chain model with state dependent arrival rates. It is accurate and computationally intensive and therefore tractable only for the networks with a small number of nodes.

Chlamtac et al. [8] have presented an efficient algorithm to optimally route lightpaths taking into consideration both the cost of using the wavelength on links and the cost of wavelength conversion in $O[(k+n) k n]$ time, where $k$ is the number of wavelengths per fiber and $n$ is the number of nodes. Barry et al. [22] have developed an analytical model to compute the blocking probabilities in circuit switched networks with and without wavelength converters. Smaller path lengths, larger switch size and larger interference length reduce blocking probabilities in networks with wavelength converters. In this paper, the link loads are not independent, however, the wavelengths used on successive links are independent of other wavelengths.

In [16], an approximate analytical model for a static routing circuit switched network of arbitrary toplogy with and without converters was established. Here, the state of successive links of the path are statistically independent and the input traffic is assumed to be Poisson. The probability of the wavelength occupancy on the fiber links are not independent. The independent link load assumption is invalid as there is a very high load correlation along the fiber links of a path in the ring. The results show that benefits of wavelength converters are modest for the ring network while the gains are significant in a large mesh torus network. The wavelength converters are effective when the network load is low and when the number of wavelengths is substantial. In an arbitrary dense WAN, the connectivity and the number of wavelengths are much more important than the availability of the wavelength converters.

Subramaniam et al. [28] have presented a probabilistic model to estimate the performance of optical networks with sparse wavelength conversion. It is shown that a small number of converters are sufficient to obtain a certain level of performance or that conversion does not offer a significant advantage. The benefits of conversion are dependent on the network load, the number of available wavelengths and the connectivity of the network. In a dense network, the effect of wavelength converters diminish and in a sparse network, calls do not mix well causing a load correlation in successive links.

In [26], an attempt is made to study the effect of wavelength conversion under dynamic non-Poisson traffic input. The model predicts that traffic peakedness plays a critical role in determining the blocking performance and the wavelength conversion is insensitivie to traffic peakedness over a large range. In [18,23] limited wavelength conversion in WDM ring networks support more load than without wavelength conversions. In [19], methods to recover from channel failures, link failures and node failures in a WDM ring network with limited wavelength conversion capabilities at the nodes are presented.

Multifiber solution as an alternative to wavelength conversion was explored in [5]. The analysis is an extension of the work in [22]. In this approach, the number of fibers to be minimized are more important than the number of wavelengths. The link utilization is lower in this approach. Mesh has higher utilization than a ring or fully connected network. In [10], limited wavelength conversion based on FWM was investigated. It assumes link load and wavelength independence. The conversion efficiency drops with increasing range. The wavelength conversion with as little as onefourth the full range gives good performance while half of the full range conversion range delivers almost all of the performance improvement. In [27], placement of wavelength converters in a path under uniform and non-uniform loads was investigated. The average blocking probability for the bus topology was derived. Sharma et al. [30] have studied limited range wavelength conversion for mesh networks over a wide range of network loads.

\section{Problem}

In the circuit switched networks, a connection request is established by configuring the wavelength routing switches at the intermediate nodes in the lightpath. Two different situations are considered in the paper. A set of requested lightpaths is assumed to be given and the problem is to realize a virtual topology by assigning a path in the physical topology for each one of the lightpaths in the set with minimum blocking probability. Since the set of lightpaths are assumed to be known a priori, the problem is called Static Lightpath Establishment (SLE). After routing the lightpaths, wavelengths are assigned to lightpaths such that each lightpath has a unique wavelength and no two lightpaths passing through a single physical link are assigned the same wavelength. Here the goals is to establish the maximum number of lightpath connections. The second case considered in the literature is one in which the lightpath requests arrive in real-time and for each incoming lightpath, a route is determined without affecting the existing lightpaths. This problem is called the Dynamic Lightpath Establishment (DLE). The lightpath is taken down after the transfer of data is completed.

\subsection{Assumptions}

- Each fiber link corresponds to a pair of unidirectional optical fibers.

- Each node can locally add or drop the optical signals. 


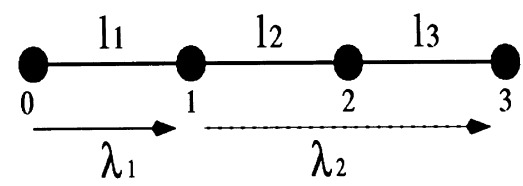

Fig. 8. Channel and wavelength converter costs.

The required number of transmitters and receivers are available at each node.

- Each routing node is a reconfigurable switch. It may have full wavelength convertibility, limited wavelength convertibility or no wavelength convertibility.

- Connection requests arrive at Poisson rate with exponential holding time. Connections requests can originate at any node and be wavelength routed to any other node.

- The lightpath connection requests that are blocked do not return.

- The network topology is static and is not reconfigured during the computation.

- Wavelength assignment for a route follows the first-fit strategy.

\subsection{Definition of the problem}

- Given the physical topology $G_{p}(V, E, C, R)$ where,

- $n_{i} \in V$ nodes of the network, $i=1,2, \ldots, N$,

- $l_{i} \in E$ represents the fiber links of the network, $i=$ $1,2, \ldots, m$,

- $c_{i} \in C$, set of converters at nodes, $i=1,2, \ldots, C$,

- $r_{i} \in R$ represents a wavelength routing switch at the nodes $i=1,2, \ldots, N$, and

- a set of wavelength channels available $\lambda_{i} \in \Lambda$ where $i=$ $1,2, \ldots, w$.

The wavelength router $r_{i} \in R$ is a reconfigurable switch and can route a wavelength on any incoming port to any outbound port. Each fiber link $l_{i} \in E$ carries at most $w$ wavelength channels. The goal is to design a virtual topology to minimize the cost and blocking probability of establishing a set of given lightpaths (SLE) or optimally route the lightpaths which arrive dynamically (DLE) to a wavelength convertible network.

\section{Analytical model}

It is computationally intractable to determine the wavelengths and conversion required at each node along a given path for a set of connection requests. The assignment of wavelengths to multihop paths is harder than the assignment of wavelengths to single-hop paths. It was proved that the wavelength assignment problem for SLE and DLE is NP complete and therefore the harder version of this problem (i.e., multihop) is also NP complete [9,11], Hence heuristics are needed to place the wavelength converters to ensure low blocking probability.

\subsection{Channel costs and conversion costs}

The route for a given connection request uses the fiber links (channel) and wavelength converters along the path. The cost of using a fiber link $l_{I}$ can be represented as $c h\left(l_{i}\right)$. The cost of using wavelength converters can be represented as $c_{i}\left(l_{i}, l_{i+1}\right)$ where $\lambda_{p}$ and $\lambda_{q}$ are wavelengths used on the links $l_{i}$ and $l_{i+1}$ respectively (Fig. 8). The channel cost $\operatorname{ch}\left(l_{i}\right)>0$ for using the wavelength $\lambda_{p}$ on link $l_{i}$. If $\lambda_{p}=$ $\lambda_{q}$ on the fiber links $l_{i}$ and $l_{i+1}$ respectively, then the wavelength converter cost is zero.

Let a source-destination $(s-d)$ connection be routed through three links $l_{1}, l_{2}, l_{3}$ on wavelengths $\lambda_{1}, \lambda_{2}$ and $\lambda_{2}$ respectively. The cost of this route is computed by the channel cost of links $l_{1}, l_{2}, l_{3}$ and the wavelength converter cost, as conversion is required from $\lambda_{1}$ to $\lambda_{2}$ at node 1 . Therefore, the total cost of this connection is given by $\operatorname{ch}\left(l_{1}\right)+c_{1}\left(l_{1}, l_{2}\right)+\operatorname{ch}\left(l_{2}\right)+\operatorname{ch}\left(l_{3}\right)$. Here $c_{1}\left(l_{1}, l_{2}\right)>0$ and $c_{2}\left(l_{2}, l_{3}\right)=0$ as wavelength on link $l_{2}$ and $l_{3}$ do not undergo any wavelength conversion.

It is necessary to minimize the overall cost of links as well as converters for a single hop or a multihop for a given set of lightpath requests or for lightpath requests arriving on real time. A route consists of an ordered sequence of links $l_{1}$, $l_{2}, \ldots, l_{p}$ with the termination of link $l_{i}$ coinciding with the beginning of the link $l_{i+1}, i=1,2, \ldots, p-1$ and a wavelength $\lambda \in \Lambda$ is used by the route on each one of the links $l_{i}$. The cost of the route which may be a single hop or a multihop path is computed by the following equation:

$C(R)=\sum_{i=1}^{p} \operatorname{ch}\left(l_{i}\right)+\sum_{i=1}^{p-1} c_{i}\left(l_{i}, l_{i+1}\right)$.

The channel cost $\operatorname{ch}\left(l_{i}\right)>0$ when a channel is used by the link $l_{i}$ and $c_{i}\left(l_{i}, l_{i+1}\right)>0$ when the wavelengths used are different on links $l_{i}$ and $l_{i+1}$ as there exists a wavelength conversion. This value is set to a large number (equal to infinity) if wavelength conversion is not possible.

\subsection{Construction of the auxiliary graph}

In the WDM optical network, each fiber link carries more than one channel unlike in an ordinary network where each physical link can be represented by a directed arc in a graph. Each fiber link represents multiple arcs in a graph, hence a modified graph known as auxiliary graph is constructed to compute the shortest path between $s-d$ pairs $[8,29]$. The auxiliary graph is constructed using the following steps:

1. Consider $V=w N$ where $V$ represents the number of vertices in the auxiliary graph, $w$ and $N$ represent the number of wavelengths and nodes of the network respectively.

2. Order the vertices in $w$ rows and $N$ columns in which 


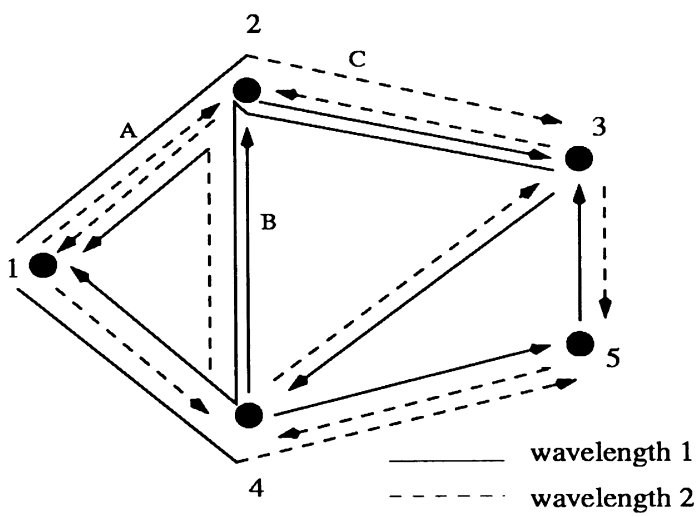

Fig. 9. A five-node network.

each column represents a node and each row represents a wavelength.

\section{For $i=1$ to $w$}

whenever there is a physical fiber link between the node $p$ and node $q$ in the network and a wavelength $\lambda_{i}$ is available, then, draw a directed arc from column $p$ to column $q$ in the auxiliary graph and label the arc with the channel cost $c h\left(l_{i}\right)$.

\section{For $j=1$ to $N$}

whenever wavelength conversion is available at a node from $\lambda_{m}$ to $\lambda_{n}$, draw a directed arc from row $m$ to $\mathrm{n}$ and label the arc with the conversion cost $c_{j}\left(l_{m}, l_{n}\right)$.

Tracing a lightpath from the source $s$ to destination $d$ can be done as follows: Each time a channel is traversed on a wavelength $\lambda_{i}$, move along the horizontal arc in the $\lambda_{i}$ plane in the auxiliary graph. Each time a wavelength conversion is performed between $\lambda_{m}$ and $\lambda_{n}$ at a node $j$, traverse the vertical edge in column $j$ from the $\lambda_{m}$ plane to $\lambda_{n}$ plane. The shortest path between two nodes $s$ and $d$ can be obtained by adding two new vertices $s^{\prime}$ and $d^{\prime}$ to the auxiliary graph such that each vertex in column $s$ is connected to $s^{\prime}$ and each vertex in column $d$ is connected to $d^{\prime}$ with zero weight edges.

\subsection{Illustrative exmaple}

Fig. 1 shows a five-node network with each fiber carrying

\begin{tabular}{c|c|c|c|c|c|}
\multicolumn{1}{c}{1} & \multicolumn{1}{c}{2} & \multicolumn{1}{c}{3} & \multicolumn{1}{c}{4} & \multicolumn{1}{c}{5} \\
\cline { 2 - 6 } 1 & 0 & 60 & 40 & 70 & 40 \\
\cline { 2 - 6 } 2 & 10 & 0 & 45 & 15 & 0 \\
\hline 3 & 40 & 20 & 0 & 80 & 40 \\
\hline 4 & 40 & 0 & 50 & 0 & 55 \\
\hline 5 & 0 & 45 & 50 & 20 & 0 \\
\hline
\end{tabular}

Fig. 10. Traffic matrix for the five-node network.

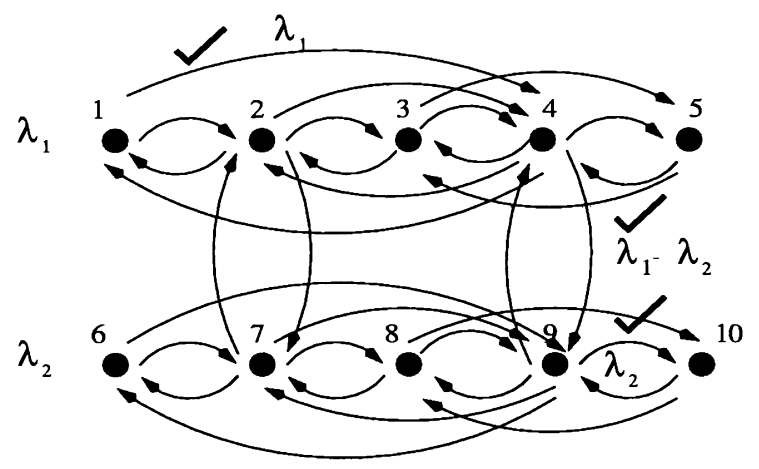

Fig. 11. Auxiliary graph.

two wavelengths $\lambda_{1}$ and $\lambda_{2}$. Each node is provided with a reconfigurable switch. In addition, wavelength converters are placed at nodes 2 and 4, which have high traffic in their output links. The lightpaths passing through different $s-d$ pairs simultaneously are shown in Fig. 9. Wavelength converter is available for each channel.

The traffic matrix for the five-node network is shown in Fig. 10. The $i j^{\text {th }}$ entry in the matrix indicate the number of connection requests that arrive between an $s-d$ pair in unit time. The traffic intensity is arranged in the descending order to ensure that higher intensity traffic nodes are allocated single-hop which results in overall reduction in delay and congestion in the network. The lightpaths in the order of descending traffic are as follows: 3-4, 1-4, 1-2, 4-5, 4-3, 5-3, 2-3, 5-2, 1-3, 3-1, 4-1, 1-5, 3-5, 5-4, 3-2, 2-4, 2-1.

The physical topology is transformed to an auxiliary graph with total number of vertices being equal to the product of the number of network nodes and the number of wavelengths. In this example, nodes $1,2,3,4,5$, are on wavelength $\lambda_{1}$ and nodes $6,7,8,9,10$, are equivalent to nodes $1,2,3,4,5$, but they are on wavelength $\lambda_{2}$. The horizontal arcs indicate that lightpaths are on the same wavelength, e.g., the lightpath between 3 and 1 is established through nodes 2 and 4 on a single hop.

The lightpath between nodes 3 and 1 passes through the reconfigurable switches at nodes 2 and 4 before reaching the destination 1 . The path between nodes 1 and 5 is established

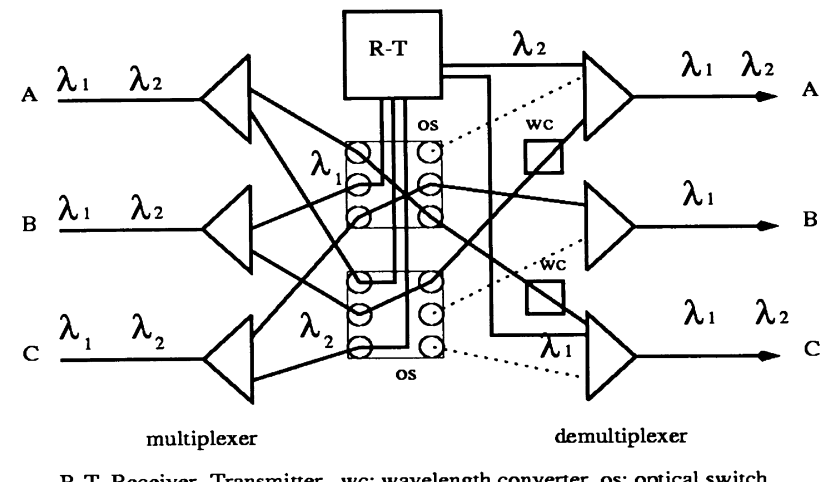

Fig. 12. Switch configuration at node 2 . 
by chaining the lightpaths between 1 and 4 on wavelength $\lambda_{1}$, converting the wavelength from $\lambda_{1}$ to $\lambda_{2}$ at node 4 and finally proceeding on wavelength $\lambda_{2}$ between the nodes 9 and 10 whose physical equivalent nodes are 4 and 5. Fig. 11 with arcs marked shows the route taken between nodes 1 and 5. The wavelengths are assigned randomly and the connection request $2-4$ is blocked. The routes for 16 connection requests are shown in Fig. 9. The auxiliary graph for the physical topology (Fig. 1) is shown in Fig. 11.

\subsection{Switch configuration}

The switch configuration at node 2 is shown in Fig. 12. Each node has provision to locally add or drop lightpaths. The node has three incoming fibers and three outgoing fibers. On the fiber $A$, signal on reaching from node 1 on $\lambda_{2}$ is dropped and a lightpath on $\lambda_{2}$ is added at this node which travels in the opposite direction.

Optical signal in the fiber $A$ arriving on $\lambda_{1}$ undergoes conversion to $\lambda_{2}$ and travels on $\lambda_{2}$ on the fiber $C$. On the fiber $B$, the signal arriving on $\lambda_{1}$ from node 4 is dropped, while the signal arriving on $\lambda_{1}$ from node 3 continues through the switch 2 without conversion to node 4 in the opposite direction. Another lightpath arriving from node 4 on $\lambda_{2}$ gets converted to $\lambda_{1}$ before proceeding on the fiber $A$. On the fiber $C$, lightpaths arriving from node 3 on $\lambda_{1}$ continues on the fiber $B$, while lightpaths arriving on $\lambda_{2}$ is dropped. Another lightpath on $\lambda_{1}$ is added between node 2 and node 3.

\section{Algorithm}

\subsection{Static lightpath establishment}

In the static lightpath establishment, a set of lightpaths requests are given a priori. The lightpath requests can be generated randomly for uniform traffic pattern or from a traffic matrix. Let the set of lightpath requests be $l p_{1}, l p_{2}$, $l p_{3}, \ldots l p_{m}$. The algorithm consists of the following phases:

- Arrange the set of lightpath requests in ascending order with respect to the length of the path.

- Place the wavelength converters at nodes having high nodal degree.

- Transform the physical link and converters into an auxiliary graph.

- Apply Dijkstra's algorithm or any shortest path algorithm to find the shortest path between $s-d$ pairs.

The phases in detail are

1. Arrange the lightpath requests set

- get the lightpath requests from the traffic matrix (based on the largest traffic node pairs or generate randomly),

- find the shortest path and path length for each one of the requests assuming unit cost for all links,
- arrange the lightpath requests in the ascending order based on the length of the paths.

\section{Placement of wavelength converters}

- given a certain number of wavelength converters, sort the nodes of network in ascending order according to their nodal degree,

- fix a threshold value for the nodal degree and allow full convertibility at the nodes above a threshold value.

3. Auxiliary graph and the cost structure

- transform the network into the auxiliary graph,

- label the arcs in the auxiliary graph with channel costs and conversion costs.

4. Find the shortest path

- consider the lightpath request,

- apply Dijkstra's algorithm to find out the minimum cost traversing from the source to the destination,

- trace back the nodes to get the minimum cost shortest path,

- map the computed path onto the network to obtain the minimum cost single-hop/multihop path,

- remove the channel arcs that constitute the path from the auxiliary graph,

- update the auxiliary graph to compute the route of the next lightpath request.

\subsection{Dynamic lightpath establishment}

In the Dynamic Lightpath Establishment, the lightpath request arrive in real time and routing is computed dynamically. The lightpath requests cannot be arranged in the order of the path lengths as they are not known a priori. The connection is taken down after the service is completed and the channel and wavelength converters will be available to new lightpath requests. The DLE consists of the following phases:

- placement of the wavelength converters at nodes having high nodal degree,

- transform the physical links and converters into an auxiliary graph,

- apply Dijkstra's algorithm or any shortest path algorithm to find the shortest path between $s-d$ pairs dynamically.

The first two phases are similar as in the static light path establishment. In the final phase, the shortest path is computed dynamically releasing the resources after the service is completed.

Find the shortest path dynamically

- consider a lightpath request arriving at time $t_{o}$. Determine whether it is a connection request or a deletion request. In the case of a connection request, go to step $\mathrm{A}$; if it is a deletion request, go to step $\mathrm{B}$, else go to step $C$. 


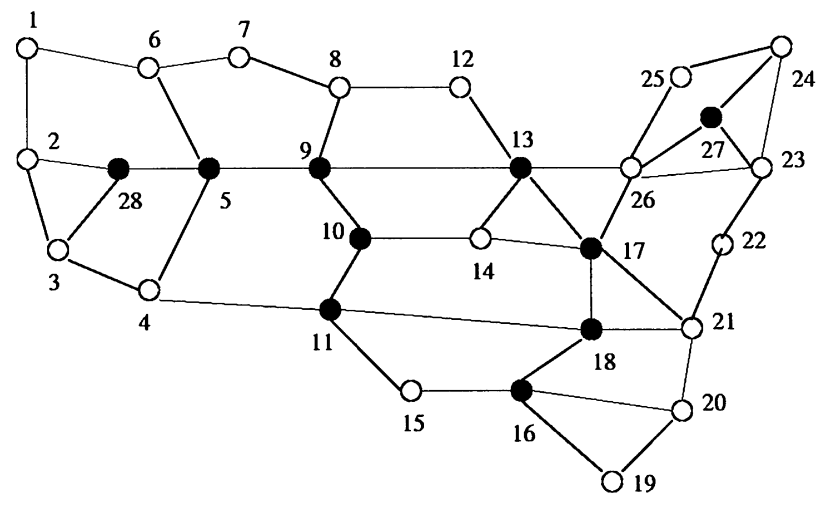

Fig. 13. 28-node USA long haul network.

1. Step A: connection request

- apply Dijkstra's algorithm to find out the minimum cost for traversing the source to each node in the auxiliary graph,

- trace back the nodes to get the minimum cost shortest path,

- map the computed path onto the network to obtain the minimum cost single-hop/multihop path.

- remove the channel arcs that constitute the path from the auxiliary graph or flag the channel arcs and converters arcs used in establishing the connection,

- update the auxiliary graph,

- fetch the next lightpath request.

2. Step B: deletion request

- retrace the path and unflag the links and converters so

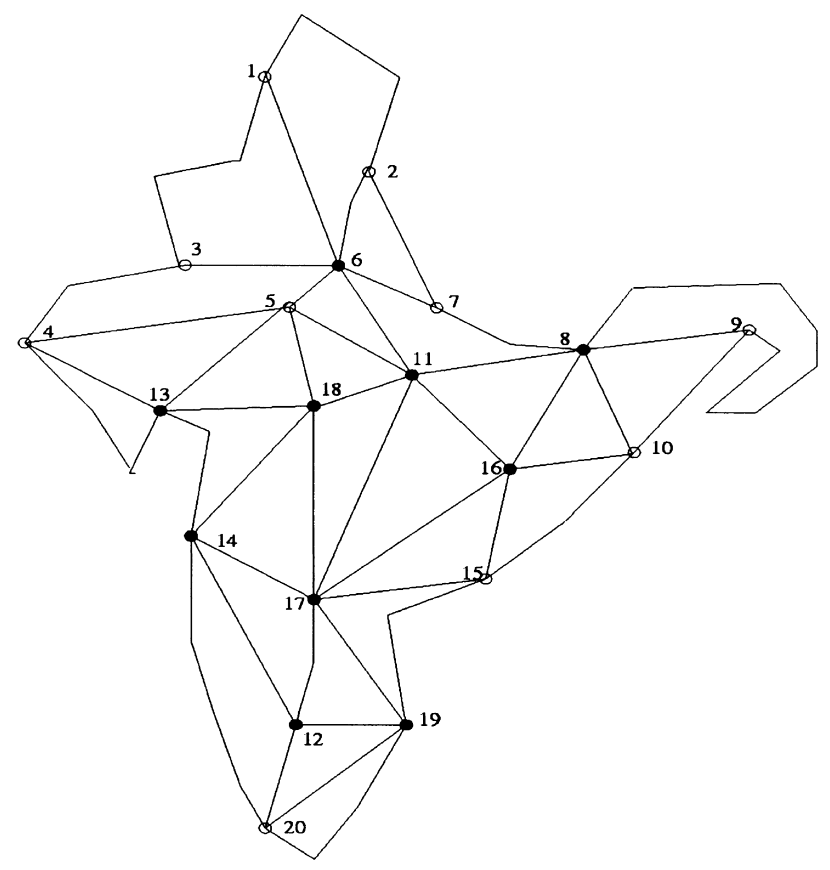

Fig. 14. 20-node INET network.

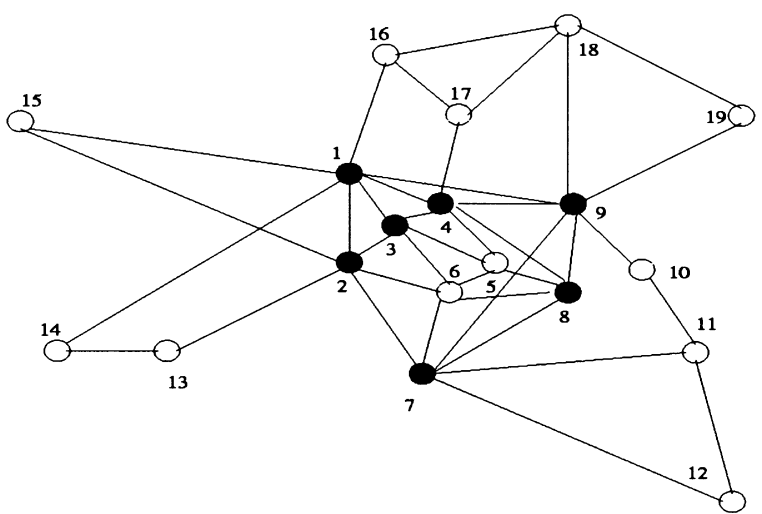

Fig. 15. 19-node European optical network.

that these resources are available for future connection request arrivals,

- fetch the next lightpath request.

\section{Step C: stop}

- this process in repeated until the required number of lightpath requests were established or the total time is elapsed.

Observation: The single-hop/multihop path between the source node $s$ and the destination node $d$ is the minimum cost path. The route will not pass through the wavelength conversion path if there is a wavelength continuous path between the source and the destination pair.

\section{Numerical results}

Simulations are carried out to evaluate the impact of wavelength converters on the blocking probability for SLE and DLE in the 28-node U.S. long haul network, 19node EON and of 20-node INET arbitrary mesh network. The connection requests are assumed to arrive according to a Poisson process. The holding times of the connection requests are exponentially distributed. The connection requests are uniformly distributed over all access station pairs. Fig. 13 shows the placement of wavelength converters at nodes with high nodal degree, i.e., at 25, 5, 9, 10, 11, 13, $16,17,18,27$ and 10 in the 28 -node network. These nodes transit a large number of paths and hence require wavelength converters.

Fig. 14 shows the placement of wavelength converters for the 20-node INET arbitrary mesh network. Wavelength Converters are placed at high nodal degrees, viz., 6, 8, 11, $12,13,16,17,18$ and 19. Similarly wavelength converters in the 19-node EON at nodes 1, 9, 7, 4, 12, 2 and 8 are shown in Fig. 15.

Four different situations are considered to study the effects wavelength converters on the blocking probability

- No convertibility at any node.

- Full convertibility at all nodes. 


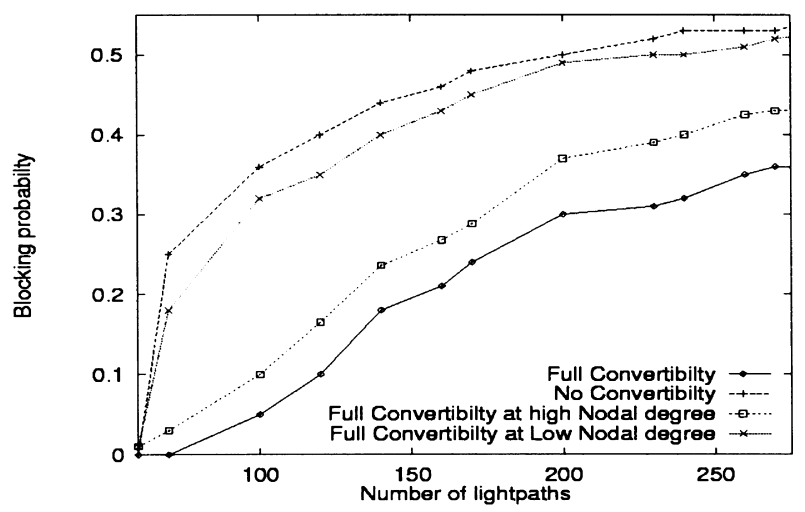

Fig. 16. Blocking probability with wavelength converters, $N=28, w=6$.

- Full convertibility at nodes with low nodal degree.

- Full convertibility at nodes with high nodal degree.

When full convertibility is introduced at all nodes, the number of wavelength converters is equal to the product of degree of the node and wavelengths per fiber link, then the network behaves like a classical circuit switched network. It is observed that full convertibility at all nodes gives the lowest blocking probability, while using no wavelength conversion at any node and full conversions at nodes with low nodal degree give almost the same performance. The best option is to place the wavelength converters at the most congested nodes or nodes with high nodal degree. These nodes are marked in the network. It gives a good trade-off between the cost and the overall blocking probability of the network. In Fig. 16, there is a rapid increase in the blocking probability initially for all cases but the blocking probability for full convertibility is lower than all other cases. Six wavelengths are assumed per fiber link.

In Fig. 17, with eight wavelengths per fiber link, the blocking probability increases with the increase in the number of static lightpath set and finally stabilizes after a certain number of lightpath set. The blocking probability reduces with the increase in the number of wavelengths and this can be observed in comparison with Fig. 16 where only four wavelengths are used per fiber link.

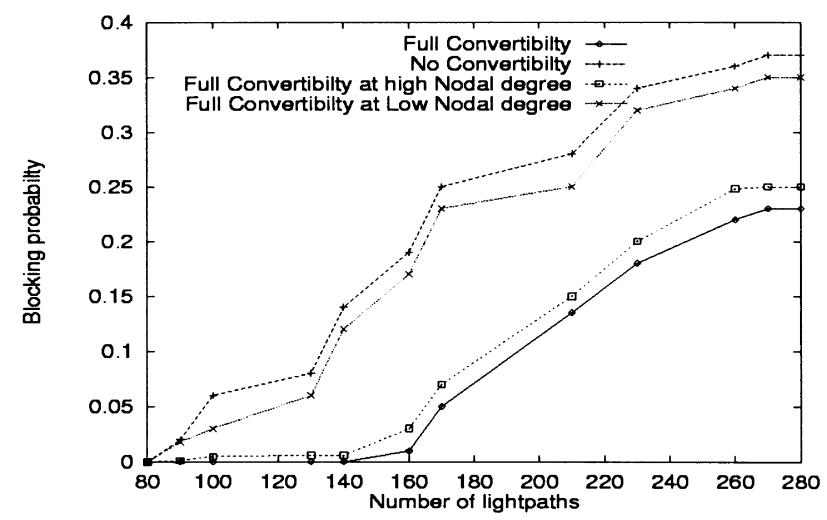

Fig. 17. Placement of wavelength converters, $N=28, w=8$.

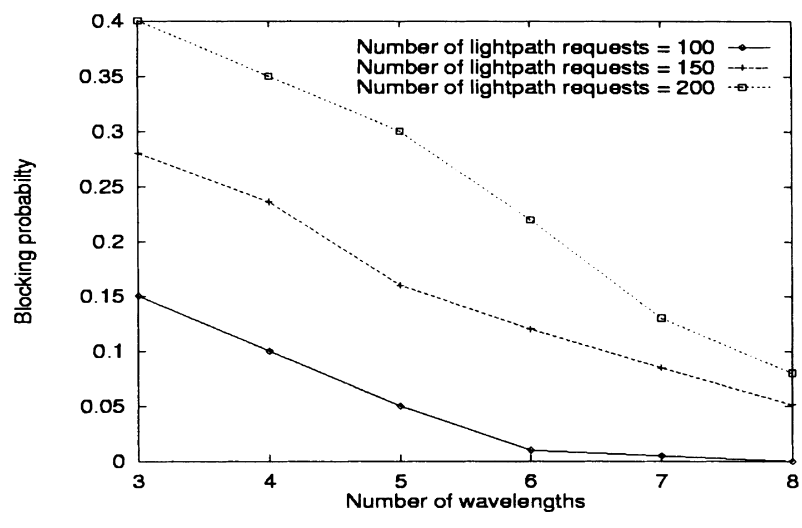

Fig. 18. Blocking probability vs. number of wavelengths, $N=28$.

An increase in the number of lightpaths requires an increase in the number of wavelengths required to maintain a given blocking probability. It has also been observed that to satisfy a given lightpath set, the number of wavelengths required reduces with the increase in the size of the network on account of the increase in the wavelength reuse. In Fig. 18, with three wavelengths per fiber link, the blocking probability is 0.15 for 100 lightpaths whereas it is as high as 0.4 when the lightpath set is 200 . For a lightpath set of 100 , the network is almost nonblocking for six wavelengths per fiber link.

In SLE, the lightpath set arranged in the ascending order of the path length results in a lower blocking probability. This is because of the establishment of a larger number of lightpaths from the given lightpath set. The shorter lightpaths use smaller number of channels and fiber links thereby allowing resources to be used by more number of lightpaths. A random static lightpath set gives better performance than the lightpath set arranged in the descending order. In Fig. 19, the blocking probability is almost the same for nearly 70 lightpath requests but blocking probability is much lower for lightpaths arranged in the ascending order for lightpath set above 70 .

In the case of DLE, the blocking probability increases with the increase in the arrival rate, but the blocking probability decreases with the increase in the number of

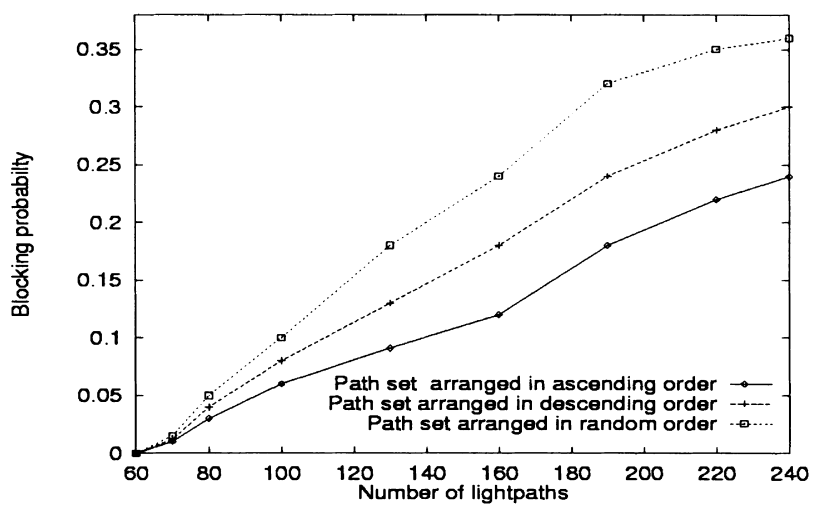

Fig. 19. Arrangement of lightpaths requests, $N=28, w=6$. 


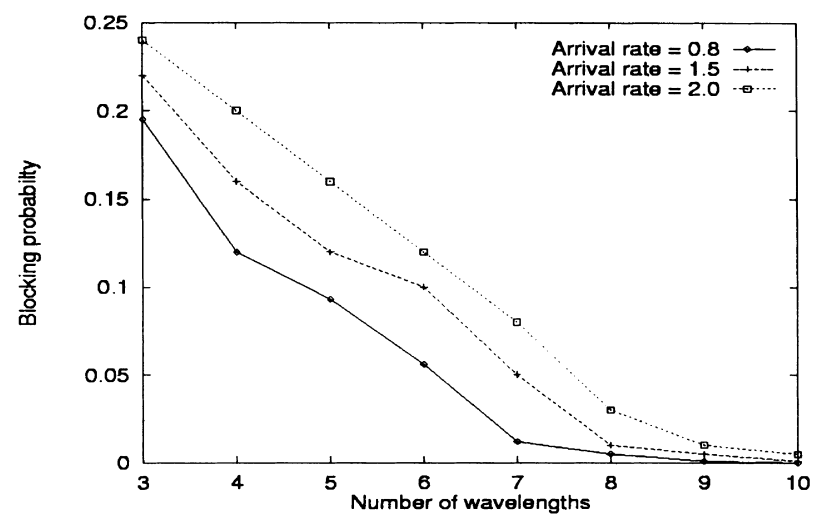

Fig. 20. Blocking probability vs wavelengths $N=28$, DLE.

available wavelengths. In Fig. 20, with three wavelengths per fiber link and an arrival rate of 2.0 per unit time, the blocking probability is 0.25 , while it is 0.2 for an arrival rate of 0.8 per unit time. With eight wavelengths per fiber link, the blocking probability is almost zero for an arrival rate of 0.8 per unit time. In Fig. 21, higher arrival rates have higher blocking probability and is in agreement with results obtained and illustrated in Fig. 20. In Fig. 22, when the arrival rate for DLE is greater than 300 lightpath requests per unit time, networks without wavelength converters perform better than networks with converters placed at low nodal degree. It indicates that at higher loads, even though less numbers of lightpaths are accepted, a large number of lightpaths with larger hop distances are supported because of the wavelength converters. With wavelength converters, long lightpaths are established which consume larger resources, resulting in the blocking of lightpaths arriving later.

The wavelength conversion gain can be defined as the ratio of the difference in the blocking probabilities without converters $P_{n w c}$ and with wavelength converters at few nodes $P_{l w c}$ and the blocking probability without wavelength converters.

$\left(P_{n w c}-P_{l w c}\right) / P_{n w c}$

At light loads, the lightpath connection requests are

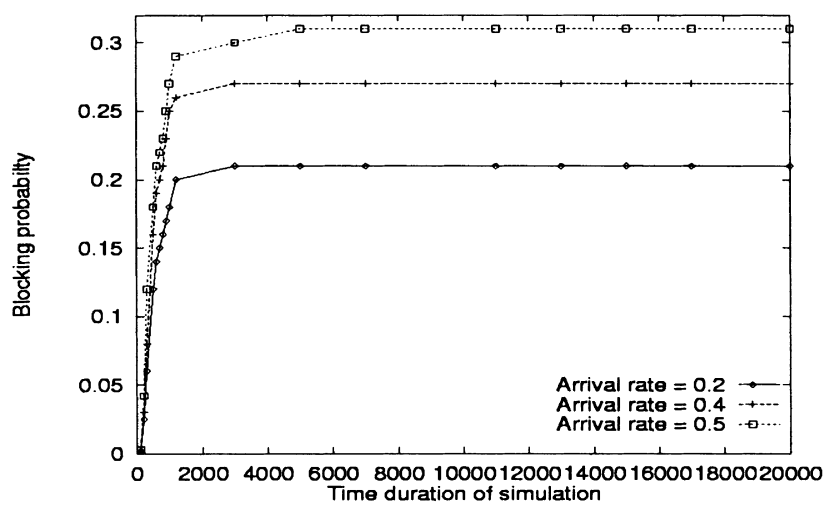

Fig. 21. Blocking probability vs time, $N=28, w=6$, DLE.

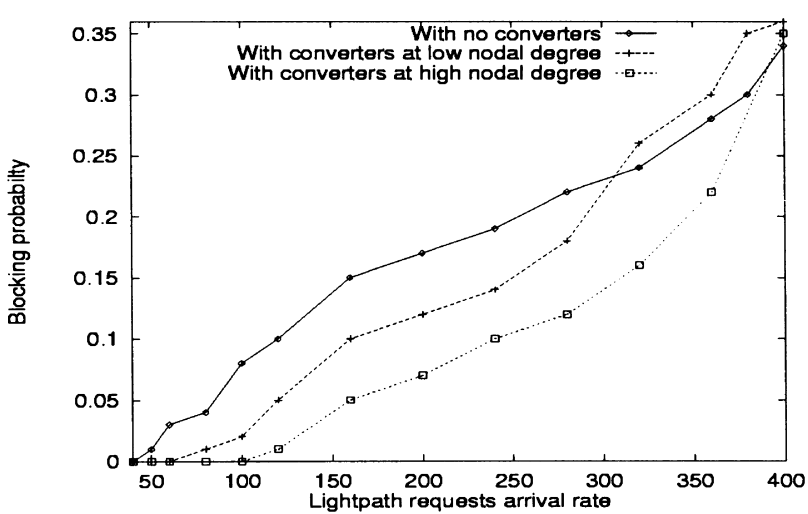

Fig. 22. Blocking probability vs arrival rate, $N=28, w=6$, DLE.

serviced without the necessity of the wavelength converters, hence the difference in blocking probability with and without wavelength converters is negligible. At medium loads, the wavelength converters increase the utility of the fiber links by overcoming the wavelength continuity constraint (by using all the wavelength channels) and the blocking probability reduces significantly. At high loads, wavelength channels on the fiber links are completely utilized and hence the difference between the blocking probabilities with and without wavelength converters is reduced.

In Fig. 23, the blocking probability gain is computed for the network with various connectivity ratio for the 12-node arbitrary network at a particular load (DLE). The maximum number of links for the 12-node mesh network is 132 . The Connectivity Ratio (CR) is defined as,

$C R=\frac{2 L}{(N)(N-1)}$

where $L$ is the number of links and $N$ is the number of nodes in the network. It can be observed that the blocking probability gain is same (zero) when the network is not connected and when the network is completely connected. The blocking probability gain when the network is not

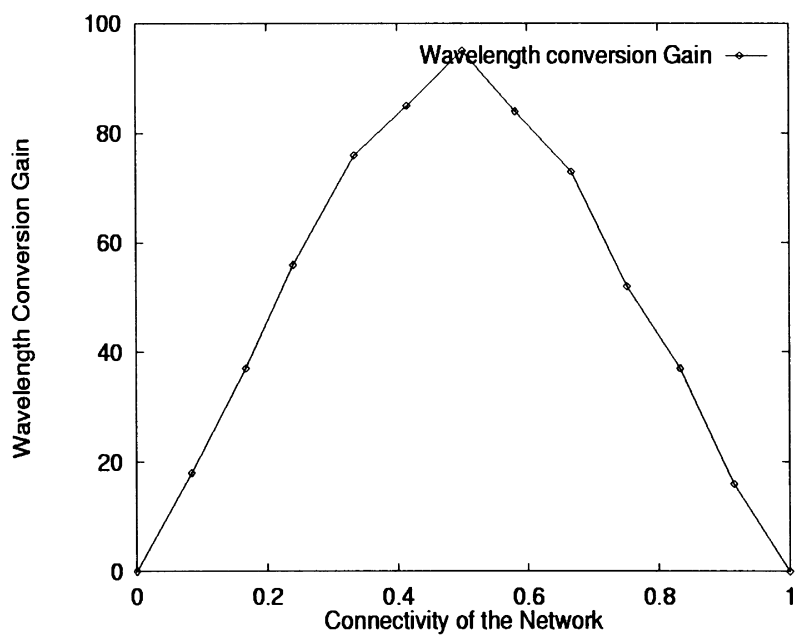

Fig. 23. Connectivity and wavelength conversion gain. 


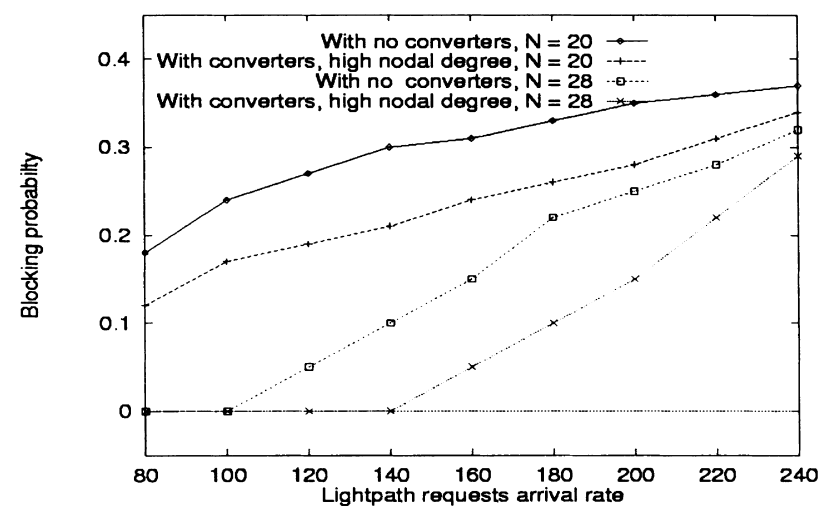

Fig. 24. Blocking probability vs network size, $w=6$, DLE.

connected is trivial and in the case of completely connected network, all the nodes are connected to all the other nodes and there are only single-hop paths and hence there is very little use of the wavelength converters. The blocking probability gain increases initially and reaches a maximum of $95 \%$ when the connectivity of the network is 0.5 and thereafter it declines reaching the lowest point for the completely connected network.

In Fig. 24, blocking probability performance is illustrated for 28-node and 20-node network with and without wavelength converters. The average degree of the nodes is four and six wavelengths are used per fiber link on both the networks. The results show that wavelength converters can improve the performance of larger networks significantly. The blocking probability is $60 \%$ lower in 28 -node networks in comparison with only $25 \%$ reduction in 20 node network at an arrival rate of 180 lightpath requests. The blocking rate is always lower in larger networks owing to higher wavelength reuse. The second reason is that the average hop distance of the lightpath requests is high in larger networks as wavelength converters accommodate longer lightpath requests.

\section{Conclusions}

Wavelength converters play an important role in circuit switched optical networks. Wavelength convertible networks reduce blocking probability, increase wavelength reuse, reduce congestion, support higher loads, ensure easier rerouting thereby increasing throughput of the network. Wavelength converters have very little impact on dense networks, but the gains are significant in sparse networks. A limited number of wavelength converters at the most congested nodes or nodes having high nodal degree give a reasonably good performance. The heuristic approach minimizes the number of converters and also reduces blocking probability in light of the high cost of the converters. The share-per-link converter reduces the switch complexity, though costlier than share-per-node converters. Lightpaths arranged in the ascending order of the path length and an increase in the number of wavelengths reduces the blocking probability in SLE. Blocking probability increases with the increase in the arrival rate for DLE. Blocking probability decreases with the increase in the size of the network. Lower blocking probability does not necessarily imply higher utilization. SLE and DLE algorithms explored and simulated in this work are efficient and cost effective. The wavelength converters, placed at the nodes with larger nodal degree perform equivalent to those networks where all nodes are provided with wavelength converters. Considering all pros and cons, the utility of limited wavelength conversions will play a significant role in the design of wavelength convertible all-optical networks.

\section{References}

[1] B. Alexander, Computing approximate blocking probabilities for a class of all-optical networks, IEEE Journal on Selected Areas in Communications 14 (5) (1996) 852-857.

[2] B. Mukherjee, Optical Networks, McGraw-Hill, New York, 1997 ISBN-0-13-178542-7.

[3] C.A. Brackett, et al., A scalable multiwavelength multihop optical network: A proposal for research on all optical networks, IEEE Journal of Lightwave Technology 11 (5/6) (1993) 736-752.

[4] C. Chen, S. Banerjee, A new model for optimal routing in all-optical networks with scalable number of wavelength converters, in: Proc. GLOBECOM 95, 1995.

[5] G. Jeong, E. Ayanoglu, Comparison of wavelength interchanging and wavelength-selective cross-connects in multi-wavelength all-optical networks, in: Proc. IEEE INFOCOMM 96, San Francisco, CA, March 1996, pp. 156-163.

[6] H. Harai, M. Murata, H. Miyahara, Performance of alternate routing methods in all-optical networks, in: Proc. IEEE INFOCOMM 97, 1997.

[7] H.G. Weber, et al., Four wave mixing for photonic switching, in: Proc. Photonics in Switching PMA5, Sendai, Japan, April 1996, pp. 160-161.

[8] I. Chlamtac, A. Farago, T. Zhang, Lightpath (wavelength) routing in large WDM networks, IEEE Journal on Selected Areas in Communications 14 (5) (1996) 909-913.

[9] I. Chlamtac, A. Ganz, G. Karmi, Lightpath communications: an approach to high bandwidth optical WANs, IEEE Transactions on Communications 40 (7) (1992) 1171-1182.

[10] J. Yates, J. Lacey, D.Everitt, M. Summerfield, Limited wavelength conversion in all-optical networks, in: Proc. IEEE INFOCOMM 96, San Francisco, CA, April 1996, pp. 954-961.

[11] K.C. Lee, V.O.K. Li, A wavelength-convertible optical network, IEEE Journal of Lightwave Technology 11 (5/6) (1993) 962-970.

[12] K.C. Lee, V.O.K. Li, Routing of all-optical networks using wavelengths outside erbium-doped fiber amplifer bandwidth, in: Proc. IEEE INFOCOMM 94, Los Angeles, CA, April 1994.

[13] K. Chan, T.P. Yum, Analysis of least congested path routing WDM lightwave networks, in: Proc. IEEE INFOCOMM 94, April 1994, pp. 962-969.

[14] K. Inoue, et al., Influence of stimulated brilliouin scattering on optimum length in fiber four wave mixing wavelength conversion, IEEE Photonic Technology Letters 7 (1995) 327-329.

[15] M.K. Rajesh, K.N. Sivarajan, Design of logical topologies: a linear formulation for wavelength routed optical networks with no wavelength changers, in: Proc. IEEE INFOCOMM 1998, April 1998.

[16] M. Kovacevic, A. Acampora, Benefits of wavelength translation in all-optical clear channel networks, IEEE Journal on Selected Areas in Communications 14 (5) (1996) 868-880. 
[17] O. Gerstel, P. Lin, G.H. Sasaki, A new angle on wavelength assignment in WDM rings: Minimize system cost, not number of wavelengths, in: Proc. IEEE INFOCOMM 98, April 1998.

[18] O. Gerstel, R. Ramaswami, G.H. Sasaki, Benefits of limited wavelength conversion in WDM ring networks, in: Proc. IEEE INFOCOMM 97, April 1997.

[19] O. Gerstel, R. Ramaswami, G.H. Sasaki, Fault tolerant multiwavelength optical rings with limited wavelength conversion, in: Proc IEEE INFOCOMM 97, April 1997.

[20] O. Gerstel, R. Ramaswami, G.H. Sasaki, Cost effective traffic grooming in WDM rings, in: Proc. IEEE INFOCOMM 98, April 1998

[21] R.A. Barry, P.A. Humblet, On the number of wavelengths and switches in all-optical networks, IEEE Transactions on Communications 42 (2-4) (1994) 868-880.

[22] R.A. Barry, P.A. Humblet, Models of blocking probability in alloptical networks with and without wavelength changers, IEEE Journal on Selected Areas in Communications 14 (5) (1996) 858-867.

[23] R. Ramaswami, G.H. Sasaki, Multiwavelngth optical neworks with limited wavelength conversion, in: Proc. IEEE INFOCOMM 97, April 1997.

[24] R. Ramaswami, K.N. Sivarajan, Routing and wavelength assignment in all-optical networks, IEEE/ACM Transactions in Networking 3 (5) (1995) 489-500.

[25] S.J.B. Yoo, Wavelength conversion techonologies for WDM network applications, IEEE Journal of Lightwave Technology 14 (1996) 955956.

[26] S. Subramaniam, A.K. Somani, M. Azizoglu, A performance model for wavelength conversion with non-Poisson traffic, in: Proc. IEEE INFOCOMM 97, April 1997.
[27] S. Subramaniam, M. Azizoglu, A.K. Somani, On the optimal placement of wavelength converters in wavelength routed networks, in: Proc. IEEE INFOCOMM 98, April 1998.

[28] S. Subramaniam, M. Azizoglu, A.K. Somani, All optical networks with sparse wavelength conversion, IEEE/ACM Transactions in Networking 4 (4) (1996) 544-557.

[29] T.E. Stern, K. Bala, S. Jiang, J. Sharony, Linear lightwave networks perfomance issues, IEEE Journal of Lightwave Technology 11 (5/6) (1993) 937-950.

[30] V. Sharma, E.A. Vavarigos, Limited wavelength translation in alloptical WDM mesh networks, in: Proc. IEEE INFOCOMM 98, April 1998.

[31] K.R. Venugopal, A. Achutharam, P. Sreenivasa Kumar, An adaptive algorithm to reduce wavelength conversion and congestion in alloptical networks, in: Proc. International Conference on Advanced Computing, Pune, India, December 1998.

[32] K.R. Venugopal, E. Ezhil Rajan, P. Sreenivasa Kumar, Performance analysis of wavelength converters in WDM wavelength routed optical networks, in: IEEE proceedings of International Conference on High Performance Computing, Chennai, India, December 1998.

[33] K.R. Venugopal, M. Shiva Kumar, P. Sreenivasa Kumar, Placement of limited range wavelength converters in wavelength routed optical networks, in: Proc. International Conference on Advanced Computing, Pune, India, December 1998.

[34] K.R. Venugopal, P. Sreenivasa Kumar, Design issues in WDM wavelength routed optical networks, in: Proc. International Conference on Advanced Computing, Chennai, India, December 1997, pp. 515-525. 